\title{
Mechanical, morphological and rheological properties of polyamide 6/organo-montmorillonite nanocomposites
}

\author{
W. S. Chow*, Z. A. Mohd Ishak \\ School of Materials and Mineral Resources Engineering, Engineering Campus, Universiti Sains Malaysia, Seri \\ Ampangan, Nibong Tebal 14300 Penang, Malaysia
}

Received 6 December 2006; accepted in revised form 24 December 2006

\begin{abstract}
Polyamide (PA6) nanocomposites containing 4 wt $\%$ organo-montmorillonite (OMMT) were melt-compounded followed by injection molding. The mechanical properties of the PA6/OMMT nanocomposites were studied through tensile and flexural tests. The rheological behaviour of the nanocomposites was determined by plate/plate rheological measurements. Attempts were made to trace the rheological parameters that reliably reflect the observed changes in the clay dispersion. X-ray diffraction (XRD) and atomic force microscopy (AFM) were used to characterize the exfoliation and dispersion of the OMMT in the PA6 matrix. The thermal properties of PA6/OMMT nanocomposite were characterized by Dynamic Mechanical Thermal Analysis (DMTA). The tensile modulus and strength of the PA6 was increased in the presence of OMMT. The flexural strength of PA6/OMMT was approximately doubled compared to the tensile strength value. The significant enhancement of both tensile and flexural strength was attributed to the delaminated clay formation. XRD and AFM results revealed the formation of PA6 nanocomposites as the OMMT was successfully exfoliated.
\end{abstract}

Keywords: polymer composites, polyamide 6, organo-montmorillonite, nanocomposites

\section{Introduction}

Polymer nanocomposites offer new technological and economical benefits. The incorporation of nanometer scale reinforcement may dramatically improve selected properties of the related polymer. These nanocomposites exhibit superior properties such as enhanced mechanical properties, reduced permeability, and improved flame retardancy [1]. Polymer layered-silicate nanocomposites are currently prepared in four ways: in-situ polymerization, solution intercalation, melt intercalation and sol-gel technology. Direct polymer melt intercalation is the most attractive because of its low cost, high productivity and compatibility with processing techniques (e.g. extrusion and injection molding) [2].

Numerous researchers described polymer-clay nanocomposites based on polyamide. Nylon 6-clay hybrid exhibits high modulus, high distortion temperature and good barrier properties of gas and water. Kojima et al. [3] have reported the synthesis of nylon 6-clay hybrid by montmorillonite intercalated with $\varepsilon$-caprolactam. They found that the silicate layers of nylon 6-clay hybrid were uniformly dispersed in the nylon 6 matrixes. Fornes et al. [4] have described the melt compounding of nylon 6/ organoclay nanocomposites by using a twin-screw extruder. Tensile modulus and strength were found to increases with increasing concentration of clay. Cho \& Paul [5] have reported that the organoclay was well exfoliated into nylon 6 matrix when compounded with twin screw extruder. Liu et al. [6] have reported the preparation of polyamide 6-clay nanocomposites/MAHgPP alloys and investigated its mechanical and dynamic mechanical properties and morphologies. The notched impact strength of 
the alloys increased remarkably while its stiffness and strength still maintained. Chiu et al. [7] have studied the effects of maleic anhydride-grafted polyolefin elastomer (POEMA) on the properties of PA6/OMMT by using melt intercalation. González et al. [8] have investigated the toughening of PA6/ OMMT by blending with maleinized styrene-ethylene/butadiene-styrene copolymer (mSEBS).

$\mathrm{X}$-ray diffraction (XRD) and transmission electron microscopy (TEM) have been adopted as the standard techniques to study the clay (or layered silicates) dispersion in polymers. X-ray diffraction permits the study of intercalation, whereas TEM could provide evidence of intercalation/flocculation and exfoliation/delamination of clay. Atomic force microscopy (AFM) is a further suitable technique to study the microstructure and nanostructure of polymer nanocomposites. Karger-Kocsis et al. [9] have studied the morphology and topography of interpenetrating vinylester/epoxy/organoclay by using AFM technique. Chow et al. [10] have investigated the blend morphology and clay dispersion in PA6/PP matrix using AFM technique. Rheometers could be used to investigate the clay dispersion in the polymer matrix. The melt-state linear viscoelastic properties for the nanocomposites are typically examined in a constant strain rheometer in either a cone and plate or parallel plate (plate-plate) geometry. According to Krishnamoorti \& Yurekli [11] the response of the intercalated and exfoliated nanocomposites to external flow is vital in their processing, but would also provide a systematic study of the response of highly anisotropic layers suspended in a viscoelastic medium. A transition from liquid-like to solid like rheological behaviour for nanocomposites at relatively low silicate loadings (1-2 vol.\%) is observed with relatively small differences between intercalated and exfoliated systems.

The present work has devoted to study the effects of 4 wt.\% OMMT on the mechanical, rheological and morphological properties of PA6. Thus, it is the aim of the present contribution to report the effect of OMMT in reinforcement and its ability of intercalation/exfoliation in PA6. This study attempts to enhance the tensile and flexural properties of PA6. Further, this paper was aimed at studying the rheological behaviour of PA6/OMMT nanocomposites at both high and low shear rates, and to interpret the rheological characteristics as a function of the morphology of the PA6 nanocomposites.

\section{Experimental}

\subsection{Specimen preparation}

The PA6 (Amilan CM 1017) used in this study was a commercial product from Toray Nylon Resin AMILAN, Japan. The melt flow index (MFI at $230^{\circ} \mathrm{C}$ and $2.16 \mathrm{~kg}$ load) and density of PA6 were $35 \mathrm{~g} / 10 \mathrm{~min}$ and $1.14 \mathrm{~g} / \mathrm{cm}^{3}$, respectively. Organomontmorillonite (Nanomer 1.30TC - OMMT) was a commercial product from Nanocor, Inc, USA. This OMMT is a white powder containing montmorillonite $(70 \mathrm{wt} \%)$ intercalated by octadecylamine (30 wt $\%)$.

Melt compounding of the PA6 nanocomposites was done on a counter-rotating twin-screw extruder (Berstoff). The extrusion zone temperature ranged from $220-230^{\circ} \mathrm{C}$. Prior to extrusion, PA6 pellets and organo-montmorillonite (OMMT) were dehumidified by using a vacuum oven at $80^{\circ} \mathrm{C}$ for $8 \mathrm{~h}$. The extrudates were pelletized with a Haake pelletizer. The pellets were injection molded into standard tensile bars using a Niigata AN 50 injection molding machine. Injection molding temperature ranged from $225-240^{\circ} \mathrm{C}$. Prior to injection molding, all pellets were dehumidified in a vacuum oven $\left(80^{\circ} \mathrm{C}\right.$ for $\left.8 \mathrm{~h}\right)$. The tensile test specimens were molded in a Type I mold according to ASTM D638.

\subsection{Mechanical studies}

Tensile tests were performed on an Instron-5582 machine at $23^{\circ} \mathrm{C}$, according to ASTM D638, at a crosshead speed of $50 \mathrm{~mm} / \mathrm{min}$. Tensile modulus, tensile strength and elongation at break were evaluated from the stress-strain data. Flexural measurements were carried out according to ASTM D790 using 3-point bending configuration at $3 \mathrm{~mm} / \mathrm{min}$ deformation rate. The support span was set at $50 \mathrm{~mm}$. Flexural modulus and strength were determined.

\subsection{X-ray Diffraction (XRD)}

Wide-angle X-ray spectra were recorded with a D 500 diffractometer (Siemens) in step scan mode using Ni-filtered $\mathrm{Cu} \mathrm{K \alpha}$ radiation $(0.1542 \mathrm{~nm}$ wavelength). Powder samples were scanned in reflection, whereas the injection-molded com- 
pounds were scanned in transmission in the interval of $2 \theta=2-10^{\circ}$. The interlayer spacing of the OMMT was derived from the peak position $\left(\mathrm{d}_{001^{-}}\right.$ reflection) in the XRD diffractograms according to the Bragg equation $(\lambda=2 d \sin \theta)$.

\subsection{Atomic Force Microscopy (AFM)}

Specimens were taken from the gauge section of the injection molded dumbbells. Their orientation was transverse to the mold flow direction. The surface of the specimens was first polished prior using further physical etching techniques. As for the physical etching, the polished surface of the specimens was eroded by $\mathrm{Ar}^{+}$ion bombardment. This occurred in a secondary neutral mass spectrometer (INA3 of Leybold) working at $500 \mathrm{eV}$ energy. The beam was focused perpendicular to the surface of the specimens. The overall ion dose was $1.9 \cdot 10^{18}$ $\mathrm{Ar}^{+} / \mathrm{cm}^{2}$. The surface profile was scanned by AFM (MultiModeTM Scanning Probe Microscope, Digital Instruments) in tapping mode and the related topography image captured.

\subsection{Plate/Plate Rheometry}

Rheological measurements were made in dynamic mode on a rheometer (ARES rheometer, Rheometric Scientific) equipped with parallel plate geometry (plate diameter: $25 \mathrm{~mm}$ ) at $230^{\circ} \mathrm{C}$. Sheets were compression molded to about $1 \mathrm{~mm}$ thickness and punched into disc of $25 \mathrm{~mm}$ diameter. Dynamic frequency scan tests were conducted for all samples at a strain sweep of $1 \%$ at $230^{\circ} \mathrm{C}$. The strain amplitude $(1 \%)$ was within the linear viscoelastic region as deduced from dynamic strain scan tests performed for PA6 and PA6/OMMT nanocomposites.

\subsection{Dynamic Mechanical Thermal Analysis (DMTA)}

The storage modulus $\left(E^{\prime}\right)$ and the mechanical loss factor $\left(\tan \delta=E^{\prime}, / E^{\prime}\right)$ as a function of temperature $(T)$, were assessed by dynamic mechanical thermal analysis (DMTA) using an Eplexor 25N device of Gabo Qualimeter, Germany. DMTA spectra were taken in tension mode at $10 \mathrm{~Hz}$ frequency in a broad temperature range $\left(T=-110 \ldots+230^{\circ} \mathrm{C}\right)$.

\section{Results and Discussion}

\subsection{Mechanical properties}

Table 1 shows the effect of OMMT on the tensile and flexural properties of PA6 nanocomposites. The tensile modulus and tensile strength of PA6 was improved significantly in the presence of OMMT. The stiffness of the OMMT filled PA6 composites is markedly higher than that of the neat PA6. This maybe attributed to the stiffness and reinforcing effects of the OMMT particles. Either full or partially delaminated clay formation is believed to be responsible for the increment in stiffness of PA6 nanocomposites. Cho \& Paul [5] reported that the modulus of PA6 composite containing $5 \mathrm{wt} . \%$ of organoclay was substantially increased relative to neat PA6. Reichert et al. [12] also showed that the tensile modulus increased as a function of organophilic layered silicates content. Shelley et al. [13] considered the possibility that the modulus improvements are due to the constraint of the polymer chains by their interaction with the clay surfaces. According to Huang et al. [14], the introduction of the montmorillonite, which has a higher modulus than the organo-soluble polyimide (PI) matrix, leads to an increase in the modulus of the nanocomposite. The tensile modulus of PI increased almost linearly up to $5 \mathrm{wt} . \%$ of montmorillonite. However, as the montmorillonite content is further increased, the aggregation of the montmorillonite leads to a leveling off or even slightly decreases in the modulus of the hybrid. The addition of organoclay yields a substantial improvement in stiffness of the composites based on PA6 [4].

Note that the flexural modulus and strength of PA6 was improved significantly in the presence of OMMT. Recall that the OMMT has a plate-like structure irrespective to its degree of exfoliation. Accordingly, the platy OMMT and its silicate layers when exfoliated are aligned in the mold flow direction (MFD), especially in the skin layers. The injection-molding direction is parallel to the long

Table 1. Effects of OMMT on the tensile and flexural properties of PA6

\begin{tabular}{|l|c|r|r|}
\hline \multicolumn{1}{|c|}{ Properties } & Unit & \multicolumn{1}{c|}{ PA6 } & PA6/OMMT \\
\hline Tensile modulus & $\mathrm{GPa}$ & $2.3 \pm 0.02$ & $3.0 \pm 0.02$ \\
\hline Tensile strength & $\mathrm{MPa}$ & $40.1 \pm 0.05$ & $68.7 \pm 0.10$ \\
\hline Elongation at break & $\%$ & $58.4 \pm 0.05$ & $3.5 \pm 0.02$ \\
\hline Flexural modulus & $\mathrm{GPa}$ & $2.1 \pm 0.02$ & $2.9 \pm 0.01$ \\
\hline Flexural strength & $\mathrm{MPa}$ & $94.2 \pm 0.10$ & $105.3 \pm 0.10$ \\
\hline
\end{tabular}


axis of the layered silicates. This forced orientation is a result of the high shear rates during injection molding. This injection molding-induced skin-core morphology causes the observed increase in the flexural strength.

\subsection{Morphological properties}

\subsubsection{X-ray diffraction (XRD)}

Figure 1 shows the XRD patterns in the range of $2 \theta=2-10^{\circ}$ for PA6, OMMT and PA6/OMMT. The XRD spectrum of the OMMT exhibits a broad intense peak at approximately $2 \theta=3.25^{\circ}$ corresponding to a basal spacing of $2.72 \mathrm{~nm}$. Note that the XRD spectra of PA6/OMMT do not shows a characteristic basal reflection of the OMMT. XRD spectra of the OMMT filled PA6 display a prominent increase in the intensity at lower $2 \theta$ values compared with neat PA6. This is due to the fact that the PA6 molecular chain success to intercalate into the inter-gallery of OMMT and further exfoliate the OMMT layered silicates. This reflects that the OMMT used was exfoliated in the PA6 matrix. Wu et al. [15] had reported a similar observation in the case of nylon 1012/clay nanocomposites. The



Figure 1. XRD spectra of PA6, OMMT and PA6/OMMT nanocomposites

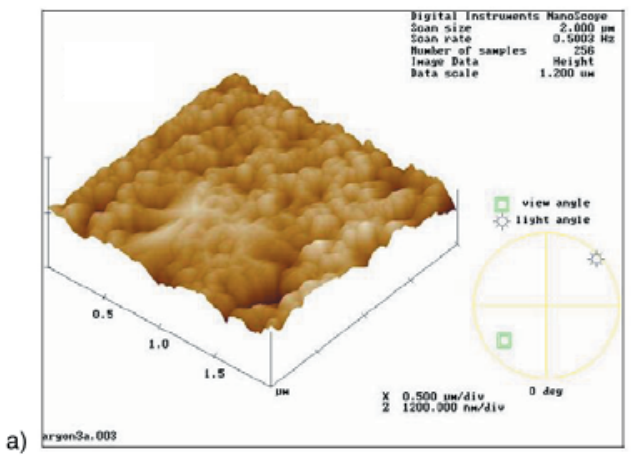

absence of the characteristic clay $\mathrm{d}_{001}$ peak indicates the exfoliation of the clay platelets in the nylon 1012 matrix.

\subsubsection{Atomic force microscopy (AFM)}

Figure $2 \mathrm{a} \& \mathrm{~b}$ display the AFM surface topography of PA6 and PA6/OMMT after Argon ion bombardment. The great difference in the topography reflects the effect of OMMT. Recall that OMMT would act as nucleant for the $\gamma$-phase of PA6 which is likely less resistant to ablation than the $\alpha$-phase. The white image represents the individual clay layers or layered silicates (i.e. platelet structure shown by the arrow). The average length of the plate-like structure is approximately 0.5 micrometer. Note that the silicate layer of OMMT was exfoliated in the PA6 matrix.

\subsection{Rheological properties}

A deeper insight into morphology-dependent flow behavior was expected from the rheological measurements performed in the viscoelastic range. The storage modulus $\left(G^{\prime}\right)$ resulting from dynamic frequency scans is depicted in Figure 3a. The storage modulus $\left(G^{\prime}\right)$ increased monotonically in the covered frequency range when OMMT was introduced to the PA6 nanocomposites. This reflects the strong effects of intercalated/exfoliated clay silicate layers on the viscosity of PA6. Besides, the increasing of the $G$ ' values of PA6/OMMT compared with the neat PA6 is attributed to the interaction of PA6 and OMMT. The slope values of $G$ ' for PA6 at $1 \mathrm{rad} / \mathrm{s}$ and $100 \mathrm{rad} / \mathrm{s}$ is 0.92 and 1.25 , respectively. On the other hand, the slope values of $G^{\prime}$ for PA6/OMMT nanocomposites at $1 \mathrm{rad} / \mathrm{s}$ and $100 \mathrm{rad} / \mathrm{s}$ is 0.89 and 0.96, respectively. According to Li et al. [16], changes of $G$ ' in the viscoelastic range sensitively

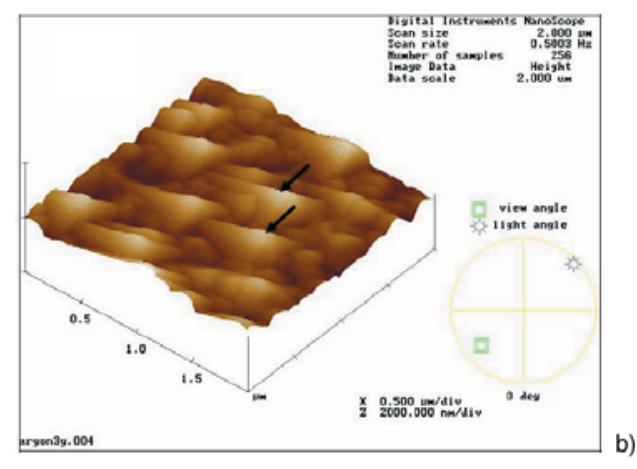

Figure 2. a - AFM surface topography of PA6; b - AFM surface topography of PA6/OMMT nanocomposites 



Figure 3. a - Variation in the storage modulus $\left(G^{\prime}\right)$ as a function of frequency for PA6 and PA6/OMMT nanocomposites; $\mathrm{b}-$ Variation in the complex viscosity $\left(\eta^{*}\right)$ as a function of frequency for PA6 and PA6/OMMT nanocomposites

reflect the effects of clay dispersion. The higher the slope, the less stable the clay is. At lower frequency (at $1 \mathrm{rad} / \mathrm{s}$ ) the polymer chains are fully relaxed and thus alterations in storage modulus $\left(G^{\prime}\right)$ and loss modulus $(G$ ") including their slopes are likely linked to the effect of the clay dispersion. This change in slope indicates that the nanocomposites attained a pseudo solid-like behaviour due to the nano-reinforcing effect of the intercalated/exfoliated clay. Similar results were reported by Hoffmann et al. [17]. The higher the $G$ ' moduli and the smaller the slope (at lower frequency), the more pronounced the interaction between the silicate platelets and their tendency to form a three-dimensional superstructure [17]. One can thus concluded that rheological characteristics $\left(G^{\prime}\right.$ and related slope) in the low frequency viscoelastic range could reflect even small changes in the clay dispersion accordingly.

Figure $3 b$ depicts the course of the complex viscosity $\left(\eta^{*}\right)$ as a function of frequency for the viscoelastic range assessed by a plate-plate rheometer. Note that the complex viscosity of PA6 increased in the presence of OMMT. The slope values of $\eta^{*}$ for PA6 at $1 \mathrm{rad} / \mathrm{s}$ and $100 \mathrm{rad} / \mathrm{s}$ are -0.04 and -0.13 , respectively. However, the slope values of $\eta^{*}$ for PA6/OMMT at $1 \mathrm{rad} / \mathrm{s}$ and $100 \mathrm{rad} / \mathrm{s}$ is -0.46 and -0.29 , respectively. According to Boucard et al. [18], at low shear rates, the silicate platelets of high aspect ratio are well separated and this strongly increases the viscosity of the melt. On the other hand, at higher shear rates the platelets are oriented in the flow direction which consequently leads to a reduction in the viscosity. One could expect that the higher the viscosity and the higher the negative slope in viscosity-frequency relation are the better is the clay exfoliation. This prediction is due to the fact that intimate clay/polymer interaction is accompanied with strong viscosity increase. However, if the clay layers are well separated from each other (exfoliated stage) then shear thinning is more probable than in case of intercalated tactoids. Recall that shear thinning presumes an alignment of the clay layer in the flow direction which occurs easily in exfoliated stage.

\subsection{Thermal analysis}

The dynamic storage modulus, as well as the tan $\delta$ versus temperature traces for the PA6 and PA6/ OMMT nanocomposites is shown in Figure $4 \mathrm{a} \& \mathrm{~b}$, respectively. It can be seen that PA6/OMMT nanocomposites exhibits higher storage modulus than PA6 (c.f. Figure 4a). This may again be attributed to the reinforcing effects of the OMMT in PA6 matrix. Figure $4 \mathrm{~b}$ shows the effect of OMMT on the loss factor $(\tan \delta)$ for PA6. Two dynamic relaxation peaks were observed at around $56^{\circ} \mathrm{C}$ and $-60^{\circ} \mathrm{C}$, which referred to as $\alpha$ and $\beta$ relaxation peaks of PA6. The $\alpha$ relaxation peak is believed to be related to the breakage of hydrogen bonding between polymer chain which induces long range segmental chain movement in the amorphous area. This is assigned to the glass transition temperature $\left(T_{g}\right)$ of PA6. Note that there is no significant changing in the $T_{g}$ value of PA6 with the incorporation of OMMT. 



Figure 4. $\mathrm{a}-E^{\prime}$ ' vs $T$ traces for PA6 and PA6/OMMT nanocomposites; $\mathrm{b}-\tan \delta$ vs $T$ traces for PA6 and PA6/OMMT nanocomposites

\section{Conclusions}

Based on this work devoted to study the effects of OMMT on the mechanical, morphological and rheological properties of PA6 nanocomposites, the following conclusions can be drawn:

(a) Modulus and strength of the PA6 was improved significantly in the presence of OMMT attributed to the stiffness, rigidity, reinforcing effects and exfoliation of OMMT.

(b) XRD and AFM techniques could be used to study the morphology of PA6/OMMT nanocomposites. The OMMT was exfoliated in the PA6 matrix. This is attributed to the strong interaction of PA6 and OMMT, and the intercalation capability of PA6 in the silicate layers of OMMT.

(c) Considering the XRD and AFM results of the OMMT dispersion in the PA6 nanocomposites, the following rheological parameters, in the viscoelastic range at low frequency, could be considered as suitable indicators: the storage modulus $\left(G^{\prime}\right)$ and its slope, and the complex viscosity $\left(\eta^{*}\right)$ and its slope. The higher $G^{\prime}$ and the smaller the related slope, as well as the higher $\eta^{*}$ and its higher related slope, the better the clay exfoliation is.

\section{Acknowledgments}

The authors would like to thank Ministry of Science, Technology and Environment (MOSTE), Malaysia for the IRPA grant and USM Short Term Grant. The authors appreciate the technical support for the AFM, DMTA and rheology tests from Professor Karger-Kocsis (Institute for Composite Materials, Kaiserslautern University of Technology, Germany).

\section{References}

[1] Ray S. S., Okamoto M.: Polymer/layered silicate nanocomposites: a review from preparation to processing. Progress in Polymer Science, 28, 1539-1641 (2003).

[2] Alexandre M., Dubois P.: Polymer-layered silicate nanocomposites: preparation, properties and uses of a new class of materials. Materials Science and Engineering: R: Reports, 28, 1-63 (2000).

[3] Kojima Y., Usuki A., Kawasumi M., Okada A., Kurauchi T., Kamigaito O.: Synthesis of nylon 6-clay hybrid by montmorillonite intercalated with $\varepsilon$-caprolactam. Journal of Polymer Science Part A-Polymer Chemistry, 31, 983-986 (1993).

[4] Fornes T. D., Yoon P. J., Keskkula H., Paul D. R.: Nylon 6 nanocomposites: the effect of matrix molecular weight. Polymer, 42, 9929-9940 (2001).

[5] Cho J. W., Paul D. R.: Nylon 6 nanocomposites by melt compounding. Polymer, 42, 1083-1094 (2001).

[6] Liu X. H., Wu Q. J., Berglund L. A., Fan J. Q., Qi Z. N.: Polyamide 6-clay nanocomposites/polypropylenegrafted-maleic anhydride alloys. Polymer, 42, 82358239 (2001).

[7] Chiu F. C., Lai S. M., Chen Y. L., Lee T. H.: Investigation on the polyamide 6/organoclay nanocomposites with or without a maleated polyolefin elastomer as a toughener. Polymer, 46, 11600-11609 (2005).

[8] González I., Eguiazábal J. I., Nazábal J.: Rubbertoughened polyamide 6/clay nanocomposites. Composites Science and Technology, 66, 1833-1843 (2006).

[9] Karger-Kocsis J., Gryshchuk O., Fröhlich J., Mülhaupt R.: Interpenetrating vinylester/epoxy resins modified with organophilic layered silicates. Composites Science and Technology, 63, 2045-2054 (2003).

[10] Chow W. S., Mohd Ishak Z. A., Karger-Kocsis J.: Atomic force microscopy study on blend morphology and clay dispersion in polyamide-6/polypropylene/ organoclay systems. Journal of Polymer Science Part B-Polymer Physics, 43, 1198- 1204 (2005). 
[11] Krishnamoorti R., Yurekli K.: Rheology of polymer layered silicate nanocomposites. Current Opinion in Colloid and Interface Science, 6, 464-470 (2001).

[12] Reichert P., Nitz H., Klinke S., Brandsch R., Thomann R., Mülhaupt R.: Poly(propylene)/organoclay nanocomposite formation: Influence of compatibilizer functionality and organoclay modification. Macromolecular Materials Engineering, 275, 8-17 (2000).

[13] Shelley J. S., Mather P. T., DeVries K. L.: Reinforcement and environmental degradation of nylon-6/clay nanocomposites. Polymer, 42, 58495858 (2001).

[14] Huang J. C., Zhu Z. K., Ma X. D., Qian X. F., Yin J.: Preparation and properties of montmorillonite/organosoluble polyimide hybrid materials prepared by a onestep approach. Journal of Materials Science, 36, 871877 (2001)
[15] Wu Z. G., Zhou C. X., Qi R. R., Zhang H. B.: Synthesis and characterization of nylon 1012/clay nanocomposites. Journal of Applied Polymer Science, 83, 2403-2410 (2002).

[16] Li J., Zhou C. X., Wang G., Zhao D. L.: Study on kinetics of polymer melt intercalation by a rheological approach. Journal of Applied Polymer Science, 89, 318-323 (2003).

[17] Hoffmann B., Kressler J., Stöppelmann G., Friedrich C., Kim G. M.: Rheology of nanocomposites based on layered silicates and polyamide-12. Colloid and Polymer Science, 278, 629-636 (2000).

[18] Boucard S., Duchet J., Gérard J. F., Prele P., Gonzalez S.: Processing of polypropylene-clay hybrids. Macromolecular Symposia, 194, 241-246 (2003). 